\title{
Some effects of early social stimulation on the emotional reactivity of ducklings
}

\author{
SANDRA H. KLEIN, HOWARD S. HOFFMAN, and PETER DePAULO \\ Bryn Mawr College, Bryn Mawr, Pennsylvania 19010
}

\begin{abstract}
Newly hatched Khaki Campbell ducklings were housed with a conspecific for $24 \mathrm{~h}$ beginning at either 1 or 5 days of age. A third group of ducklings received no social stimulation. When tested on Day 7, ducklings afforded early social stimulation exhibited less distress calling and crouching in a novel open field than ducklings exposed to late or no social stimulation. A second experiment asked whether the above result was due to early social stimulation per se or to the separation which occurred at its termination. Newly hatched ducklings were housed with a conspecific beginning on Day 1 . One-half of the birds were separated from their companions on Day 2; the rest were separated on Day 6. On Day 7, the ducklings that were separated early exhibited less crouching and distress calling in a novel open field than the birds that were separated late. This finding implies that the withdrawal of social stimulation, rather than social stimulation per se, was responsible for the reduced emotional behavior observed in Experiment I. It is concluded that early withdrawal of social stimulation generated a strong aversive reaction and that it was the strength and timing of this reaction that mediated the emotionality moderating effects found here. Under these circumstances, the primary function of early social stimulation was to establish a condition whereby the aversive reaction generated by separation would occur early.
\end{abstract}

A number of studies have revealed that animals stimulated by stressful electrical shock in infancy exhibit reduced emotional behavior when they are tested at a later age in an open field or other fearprovoking situation (Denenberg, Carlson, \& Stephen, 1962; Lindholm, 1962). In these studies, the subjects were rats or mice, but similar early stimulation effects have recently been obtained in ducklings (Ratner, in press). Ratner found that when ducklings were shocked soon after hatching they showed less distress calling and crouching in a subsequent openfield test than ducklings that were either shocked at a later age or were never shocked.

The research reported here was designed to elaborate on Ratner's work by asking whether the emotionality moderating effects of early stimulation in ducklings were limited to forms of stimulation that, like electrical shock, are clearly aversive. In particular, the work asked whether or not the social stimulation provided by early exposure to a conspecific might not also engender reduced emotional behavior when subjects were subsequently tested in an open field. That the stimulation provided by a conspecific is not aversive to the very young duckling seems clear from the finding that they immediately approach and stay near a conspecific when first

This research was supported by Grant MH 19715 from the National Institute of Mental Health, directed by H. S. Hoffman. Requests for reprints should be addressed to Howard S. Hoffman, Department of Psychology, Dalton Hall, Bryn Mawr College, Bryn Mawr, Pennsylvania 19010. exposed to it and that they can be readily trained to peck a key when the only reinforcing event consists of a brief opportunity to view another duckling (Stratton, 1971). In the initial study reported here, otherwise isolated ducklings were permitted $24 \mathrm{~h}$ of exposure to another duckling beginnin's on Day 1 posthatch or on Day 5 posthatch. The open-field behavior of these subjects was then compared to the open-field behavior of a third group of ducklings that had never previously been exposed to conspecifics.

\section{EXPERIMENT I}

Subjects

\section{Method}

The subjects were 39 Khaki Campbell ducklings (Anas platyrhynchos domesticus) that were hatched from eggs obtained from George F. Shaw, Inc., West Chester, Pennsylvania.

The ducklings were hatched in visual isolation and, except as otherwise noted, were maintained in separate housing units. Each unit consisted of a 56.7-liter translucent plastic comainer that was lined with a disposable polyethylene bag partially filled with absorbent bedding material (San-i-cel). Food and water were available at all times within each housing unit. All housing units were kept in a single room, thus the subject (or subjects) in any one unit could hear but could not see ducklings in other units.

\footnotetext{
Apparatus

The open-field test was conducted in a $47-\mathrm{cm}$-high box, the floor of which measured $71 \times 46 \mathrm{~cm}$. During the open-field test, distress calls were detected by a specially constructed voice key which, through selective filtering, was sensitive only to those sounds in the frequency range within which distress vocalizations typically fall (approximately $3,000-4,000 \mathrm{~Hz}$ ). In addition, the
} 


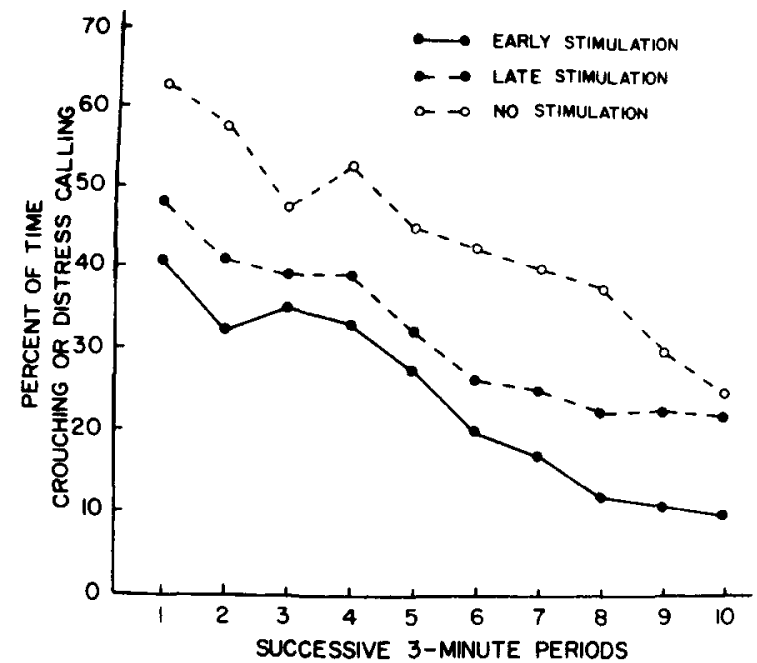

Figure 1. The mean percent of time that ducklings in the early-, late-, and no-stimulation groups crouched or distress-called during successive 3-min periods in the open field.

ducklings' behavior in the open field was continually monitored through a closed-circuit television system.

\section{Procedure \\ Phase I: Stimulation. When the ducklings were approximately $8 \mathrm{~h}$ old, they were removed from the incubator and randomly assigned to one of three equal-sized groups $(N=13)$ : "early stimulation," "late stimulation," or "no stimulation." Subjects in the no-stımulation group were placed in individual housing units, where they remained until testing occurred. Subjects in the early-stimulation group were initially housed in pairs, but due to the uneven number of subjects, it was necessary to place three (rather than two) subjects in one of the housing units. Sub- jects in the early-stimulation group remained together for $24 \mathrm{~h}$ (i.e., untıl they were $32 \mathrm{~h}$ old). At that time, they were separated and placed in individual housing units until testing ensued. Late- slimulation subjects, like no-stimulation subjects, were placed in individual housing units at $8 \mathrm{~h}$ posthatch. However, where the no- stimulation subjects were never parred, the late-stimulation birds were paired for $24 \mathrm{~h}$ beginning on Day 5 . At the end of this period, they were separated and once more housed individually. (Again, because of the odd number of subjects in this group, it was necessary to place three subjects in one of the units.) \\ Phase 2: Open-field test. On the 7th day posthatch, each duckling was individually placed in the open field for $30 \mathrm{~min}$ and records of distress calling and crouching were obtained. Distress calls were automatically recorded by the detection unit described earlier. The tendency to crouch was assessed by an observer who monitored the duckling's behavior via the closed-circuit television system. To prevent possible recording bias during the assessment of crouching, the observer was not informed as to the group to which a given duckling belonged.}

\section{Results}

Birds in the no-stimulation condition were rarely observed to emit any distress calls in their housing units, but both early- and late-stimulation ducklings emitted many distress calls (when they were first separated from their companions), and this behavior usually persisted for several hours. When placed in the open field, all subjects emitted distress calls, but for some birds this behavior was preceded by a period of crouch $n$. Since there were no instances of a duckling's simultaneously distress-calling and crouching in the open field, the time spent in the two behaviors was combined to derive a single index of a given duckling's emotional behavior during the openfield test.

Figure 1 shows the mean percent of time that subjects in each of the three groups showed distress calling or crouching during successive 3-min periods of this test. Early-stimulation ducklings showed less of these behaviors than late-stimulation or no-stimulation subjects. The late-stimulation birds showed an amount of distress calling and crouching that was intermediate between that shown by the other two groups. A two-factor repeated measures analysis of variance revealed a significant groups effect, $F(2,36)$ $=3.28, \mathrm{p}<.05$, and a significant periods effect, $F(9,324)=15.1, p<.05$. The Groups by Periods interaction was not significant, $F(18,324)=.39$. A Newman-Keuls analysis for individual groups differences (Winer, 1962) showed that all three groups were significantly different from each other in the percent of time distress-calling or crouching ( $p<.05$ ).

\section{Discussion}

These findings make it clear that some aspect of the sequence of events involved in a day of exposure to a conspecific can moderate a duckling's subsequent expression of emotional behavior. Moreover, the magnitude of the effect is importantly determined by when this exposure occurs. Early exposure produces a larger reduction in emotional behavior than does later exposure. While this finding lends credence to the hypothesis that the early stimulation effect can be obtained with stimulation that is not itself aversive, the procedures of Experiment $I$ involved the withdrawal of social stimulation, an event which invariably led to distress calling. This raises the question as to whether the earlystimulation effect obtained here was due to social stimulation per se or to the aversive consequences engendered by withdrawing that stimulation. Experiment II was designed to answer this question.

\section{EXPERIMENT II}

In Experiment II, otherwise isolated ducklings were initially exposed to a conspecific on Day 1 and separated from it on either Day 2 or Day 6. As in Experiment I, subjects were subsequently tested for crouching and distress calling on Day 7 . If the reduction in emotionality obtained in Experiment I was due to social stimulation per se, then the ducklings separated on Day 6 in Experiment II might show even less emotionality than those separated on Day 2 , since the former ducklings will have had more social stimulation. On the other hand, if the 
effect was due to withdrawal of that stimulation, then the ducklings separated early (on Day 2) should show less emotionality than the ducklings separated late (on Day 6).

\section{Method}

Subjects

The subjects were 27 ducklings hatched and housed as in Experiment I.

\begin{abstract}
Apparatus
The open-field apparatus described in Experiment I was also used in the present study.

\section{Procedure}

Phase 1: Stimulation. At $8 \mathrm{~h}$ posthatch, the ducklings were removed from the incubator and pairs of birds were placed in each of a number of housing units. (Due to the uneven number of subjects, three birds were placed in one of the units.) All subjects in a given unit were then randomly assigned to either an "early. separation" or a "late-separation" condition. The 14 subjects in the early-separation condition were moved to individual housing units at $32 \mathrm{~h}$ posthatch. The 13 late-separation ducklings remained together until Day 6 posthatch, when they too were moved to individual housing units.

Phase 2: Open-field test. On Day 7 posthatch, each duckling was individually placed in the open field for $30 \mathrm{~min}$. Distress vocalizations as well as crouching behaviors were monitored as in Experiment I.
\end{abstract}

\section{Results}

In this study, as in Experiment $\mathrm{I}$, all subjects were observed to distress-call when they were separated from their partners-indicating that the separation was aversive. A single index of each duckling's behavior in the open field was calculated as in Experiment I, using percent of time distress-calling and crouching during successive 3 -min periods of the test. Figure 2 shows that the early-separation birds displayed these behaviors a smaller percent of the time than the late-separation birds. An analysis of variance again revealed a significant groups effect, $F(1,25)=8.77, p<.01$, a significant periods effect, $F(9,225)=13.83, p<.01$, and a nonsignificant interaction, $\mathrm{F}(9,225)=.14, \mathrm{p}>.05$.

Comparisons of the results of Experiments I and II were not made because different hatches of ducks were involved. Similar studies in our laboratory have revealed that different hatches may show quite different overall levels of emotionality. This precludes comparisons between hatches, but does not preclude comparisons between groups of randomly assigned subjects within hatches.

\section{DISCUSSION}

The results of Experiment II, considered in combination with those of Experiment $\mathrm{I}$, indicate that early separation from a conspecific, rather than early social stimulation per se, reduces subsequent distress calling and crouching in the open field. Both

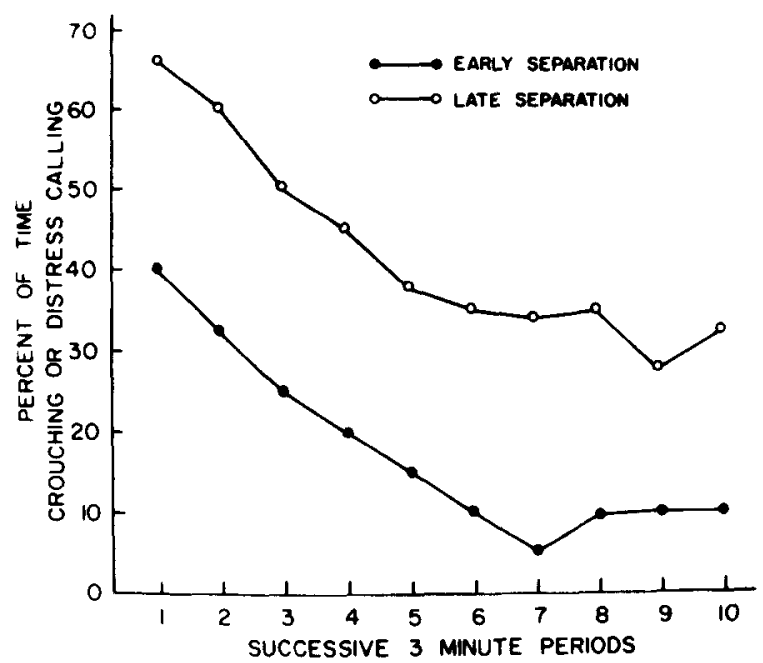

Figure 2. The mean percent of time that ducklings in the earlyand late-separation groups crouched or distress-called during successive 3 -min periods in the open field.

groups in Experiment II experienced early social stimulation, but the group separated on Day 2 showed less emotionality than the group separated on Day 6. Clearly, this finding provides no comfort for our original hypothesis that early social stimulation would itself reduce subsequent emotional behavior. If social stimulation per se were the critical factor, then the latter group, which received even more social stimulation, should have shown as little emotionality as those separated on Diy 2 or, perhaps, even less.

An explanation for why the withdrawal of social stimulation should be aversive and elicit distress calling is suggested by a consideration of the opponent-process motivation theory initially formulated by Solomon and Corbitt (1973) and applied to imprinting by Hoffman and Solomon (1974). According to the opponent-process theory, the onset and maintenance of any affect-arousing stimulus (whether positive or negative in hedonic quality) creates a primary motivational condition called the A process. The occurrence of the A process then automatically arouses an affective process (B process) which is opposite in hedonic quality to that generated by the A process. When the affect-arousing stimulus is removed, A process dissipates rapidly but B process dissipates only sluggishly. Consequently, an affective state ensues which is opposite to the affective state generated by stimulus presentation.

In terms of the situation described here, early exposure to a conspecific presumably creates an $\mathbf{A}$ process that is hedonically positive or pleasurable. This A process is opposed by a hedonically negative or aversive B process which manifests itself when the social stimulation provided by the conspecific is withdrawn. This hedonically aversive motivational after- 
effect does not develop in ducklings that are never exposed to a conspecific, since no A process is created in these birds. Thus, the no-stimulation birds of Experiment I should not have experienced aversive stimulation from a B process. According to this interpretation, the aversive experience is not the absence of social stimulation per se but, rather, the withdrawal of social stimulation. Support for this interpretation has been provided by a study in which ducklings' distress calling was monitored on the first day after hatching in the environment in which the hatching occurred (Hoffman \& Ratner, 1973). Little or no distress calling was recorded in the first $17 \mathrm{~h}$ posthatch. These ducklings were then exposed to a moving (imprinting) stimulus for $10 \mathrm{~min}$. At such an early age, a novel moving stimulus evokes filial rather than fear-type reactions, and these ducklings continued to emit little or no distress calls in its presence. Within seconds after the stimulus was withdrawn, however, high levels of distress calling ensued. Evidently, the aversive reaction was a product of the presentation and subsequent withdrawal of the imprinting stimulus rather than the absence of the stimulus per se. Similarly, in the present study, the no-stimulation birds were seldom observed to distress-call in their housing units, but ducklings in the other experimental groups emitted distress calls for hours upon separation from a conspecific. This leads to the conclusion that the reduced emotional behavior of the ducklings that were both stimulated and separated early was a product of the strength and timing of the aversive state that occurred following separation. This leads to the further conclusion that, in the present context, the primary function of early stimulation was to establish a condition under which the aversive state generated by separation would occur early.

\section{REFERENCES}

Denenberg, V. H., Carlson, P. V.. \& Stephen, M. W. Effects of infantlle shock upon emotionalsty at weaning. Journal of Comparative and Physiological Psychology, 1962, 55, 819-820.

Hoffman, H. S., \& Ratner, A. M. Effects of stimulus and environmental familiarity on visual imprinting in newly hatched ducklings. Journal of Comparative and Physiological Psychology, 1973, 85, 11-19.

Hoffman. H. S.. \& Solomon, R. L. An opponent-process theory of motivation: III. Some aftective dynamics in imprinting. Learming and Motivation, 1974, 5. 149-164.

LinDHolm. A. Critical periods and the effects of early shock on later emotional behavior in the white rat Journal of Comparative and Physiological Psychology, 1962, 55, 597-599.

Ratner. A. M. The effects of aversive stimulation in the context of imprinting. Journal of Expenmental Psychology' Animal Behavior Processes, in press.

Solomon, R. L., \& Corbirt, J. D. An opponent-process theory of motivation: II. Cigarette addiction. Journal of Abnormal Psychology, 1973, 81, 158-171.

Stratton. V. N. The control of social behavior in young ducklings. Unpublished dissertation. Pennsylvania State University, Department of Psychology, 1971.

WINER, B. J. Statistical principles in experimental design. New York: McGraw-Hill, 1962.

(Received tor publication September 22, 1975; revision accepted December 3, 1975.) 